\title{
Predator diet, origin or both? Factors determining responses of omnivorous amphipods to predation cues
}

\author{
Łukasz Jermacz $\mathbb{1} \cdot$ Anna Dzierżyńska-Białończyk · Jarosław Kobak
}

Received: 29 March 2016/Revised: 21 June 2016/Accepted: 8 July 2016/Published online: 5 August 2016

(C) The Author(s) 2016. This article is published with open access at Springerlink.com

\begin{abstract}
Detection of chemical cues of predators and food resources is a key for the behaviour of many species in aquatic ecosystems. We checked whether predator origin and diet, containing potential food and/ or alarm signals, affect the behaviour of omnivorous prey partly sharing their diet with a top predator. We conducted y-maze experiments to study the responses of invasive omnivorous and cannibalistic amphipods (Dikerogammarus villosus and Pontogammarus robustoides) to chemical cues of fish predators, injured amphipods and chironomid larvae (common amphipod food). As the predators, we used the goby Babka gymnotrachelus (sympatric with the amphipods) and piranha Pygocentrus nattereri (allopatric to the amphipods). The fish were either starved or fed amphipods or chironomids. D. villosus preferred predators fed chironomids and conspecifics as well as crushed conspecifics and chironomids, but avoided both hungry predators. Thus, D. villosus may perceive the presence of a top predator as an indication of both
\end{abstract}

Handling editor: André Padial

Electronic supplementary material The online version of this article (doi:10.1007/s10750-016-2917-1) contains supplementary material, which is available to authorized users.

Ł. Jermacz $(\bowtie) \cdot$ A. Dzierżyńska-Białończyk · J. Kobak Department of Invertebrate Zoology, Faculty of Biology and Environmental Protection, Nicolaus Copernicus University, Lwowska 1, 87-100 Toruń, Poland e-mail: lukasjermacz@gmail.com food resource and predation risk. $P$. robustoides avoided predators fed both amphipods (particularly conspecifics) and their alarm substances. The responses of both species depended more on the predator diet than on its species, which is likely to facilitate the recognition of allopatric predators and survival in newly invaded areas.

Keywords Alarm substance $\cdot$ Anti-predator behaviour - Dikerogammarus villosus .

Pontogammarus robustoides · Predation risk assessment . Predator kairomone

\section{Introduction}

Predation is one of the strongest factors shaping the relations between animals from successive trophic levels. As a consequence of predation pressure, prey species exhibit multilevel defence strategies expressed in different aspects, such as changes in behaviour, morphology or life history, taking place at an individual or population level (Sih \& Wooster, 1994; Weber, 2003). Exposure to predator induces sustained psychological stress (Clinchy et al., 2013), which results in energetically costly non-consumptive effects (Werner \& Peacor, 2003), consisting in decreased foraging times, occupation of less suitable habitats, reduced fecundity (Gliwicz, 1994) and potential exposure to another predator (Relyea, 2008). High costs of anti-predator behaviour are also associated 
with incorrect prey responses to non-predators or nonactive predators, which result in wasting valuable time and energy that would otherwise be available for foraging and reproduction (Lima \& Dill, 1990; Dunn et al., 2008). Incorrect identification of predation risk (type of predator) may also be responsible for an ineffective defence strategy (Åbjörnsson et al., 2004; Banks \& Dickman, 2007). That is why a proper assessment of predation risk using all available signals indicating the presence of a predator, its condition and probability of attack, including feeding strategy (Bernot \& Turner, 2001; Haddaway et al., 2014), size (Radloff \& Du Toit, 2004), density (Pennuto \& Keppler, 2008) and hunger level (Åbjörnsson et al., 1997 ) is crucial for prey individuals. Aquatic organisms often use chemical signals for communication (Brönmark \& Hansson, 2000; Beermann et al., 2015). These include kairomones: substances released by living organisms, which are perceived by and benefiting individuals of another species (Brönmark \& Hansson, 2000). Kairomones are commonly involved in prey-predator recognition systems (Baumgärtner et al., 2003; Schoeppner \& Relyea, 2009; Szokoli et al., 2015).

For omnivorous and cannibalistic prey species, for example, Gammarus spp. (MacNeil et al., 1997), exudates of a top predator and/or conspecific alarm substances may contain ambiguous information not only on the predation risk, but also on the vicinity of a potential food source, as postulated (though not demonstrated) by Paterson et al. (2013). This phenomenon, however, has not been fully confirmed by experimental studies yet. In such cases, ecological stress induced by predators (Clinchy et al., 2013), responsible for non-consumptive predator effects, might be reduced compared to that posed by the scent of a predator alone.

In a stable ecosystem shaped by long-term evolutionary processes, the relationship between predator and prey is generally stable, modulated by slight seasonal fluctuations. However, this situation can be changed when non-native species with no common evolutionary history are involved. Invasive species can be more susceptible prey to a native predator (Zuharah \& Lester, 2010). One of the fundamental problems encountered by non-native organisms on newly inhabited territories is the presence of unknown predators against which no defence mechanisms had evolved (the "naïve prey" hypothesis; Sih et al. 2010).
Thus, the ability of a rapid adaptation to a new environment (Yoshida et al., 2003) including novel predators (Chivers et al., 1996; Wisenden et al., 1997) can be crucial for the success of non-native species on a new territory. Evolutionary changes in anti-predatory responses can be very fast and take place within just a few generations (Yoshida et al., 2003). However, recent evidence suggests that a lack of a common evolutionary history not always corresponds to the lack of appropriate anti-predator responses (e.g. Haddaway et al., 2014). There may be several reasons for such an appropriate response to a novel predator (Bourdeau et al., 2013). For example, a non-native predator may emit a similar cue as those produced by co-existing predators (Sih et al., 2010) or prey may recognize the alarm substances of consumed or digested conspecifics in predator exudates (Chivers \& Smith, 1998).

To check whether a predator diet (containing conspecific or heterospecific alarm cues) and origin (co-evolving with prey or allopatric) affect antipredatory responses of omnivorous prey, we tested the behaviour of two invasive Ponto-Caspian amphipods: Dikerogammarus villosus (Sowinsky, 1894) and Pontogammarus robustoides (G.O. Sars, 1894) in $y$-maze experiments in the presence of sympatric or allopatric predators fed different types of food. These species belong to the most successful invaders in European waters (Grabowski et al., 2007; Rewicz et al., 2014). D. villosus is one of the most widespread amphipods in Europe, reaching France and Great Britain in the West and exerting a strong impact on local communities (Rewicz et al., 2014). The distribution of $P$. robustoides is more limited, with eastern Germany being its westernmost location, perhaps due to its avoidance of flowing waters (Jażdżewski et al., 2002).

We hypothesized that for omnivorous and cannibalistic amphipods (Dick \& Platvoet, 2000; BacelaSpychalska \& van der Velde, 2013), exudates of predators fed particular types of diets might also contain attractants indicating the increased availability of food resources (Wisenden et al., 2009; Paterson et al., 2013). This would reveal a dual nature of a predator cue, as information on both potential danger and availability of food. Additionally, the ability to detect conspecific and/or heterospecific alarm cues in predator exudates might allow prey to respond flexibly to unknown predators (Chivers \& Smith, 1998) and/or 
limit their responses only to the presence of a currently foraging predator. Therefore, we expected that the anti-predator behaviour of invasive amphipods would depend on the composition of the predator diet, containing amphipod alarm substances or not.

\section{Methods}

Origin and maintenance of animals

We used amphipods collected in the littoral zone of the Włocławek Reservoir (a dam reservoir on the lower River Vistula, Central Poland), N: $52^{\circ} 37^{\prime} 03^{\prime \prime}$, E: $19^{\circ} 19^{\prime} 37^{\prime \prime}$. P. robustoides was captured with hand nets from the sandy bottom of the reservoir at a depth of 0.1-0.3 m. D. villosus was obtained using artificial substrata made of plastic Christmas tree branches submerged at a depth of $0.5 \mathrm{~m}$. The amphipods were kept in 100-L single species stock tanks in water of the same physicochemical parameters as during the experiments (temperature $19^{\circ} \mathrm{C}$, sustained by air conditioning, conductivity $480-530 \mu \mathrm{S} / \mathrm{cm}$ ). We checked the water quality parameters using a multimeter Multi340i (WTW GmbH, Weilheim, Germany). The tank bottoms were covered by sand and zebra mussels providing shelters for the animals. Each individual was used in the experiments only once, not earlier than 5 days and not later than 2 weeks after capture, to allow their acclimation to laboratory conditions and to avoid exhaustion by prolonged captivity. In preliminary observations, we did not observe any changes in amphipod behaviour during that period.

We used two predator species: the Ponto-Caspian racer goby Babka gymnotrachelus (Kessler, 1857), sympatric to the selected amphipods, expanding its range in Europe together with them and including them in their diet (Kakareko et al., 2005; Brandner et al., 2013), as well as the red piranha Pygocentrus nattereri, a species with no common evolutionary history with the tested amphipods. Therefore, we could test gammarid responses to familiar predators as well as to those they meet for the first time. The racer goby individuals were collected by electrofishing (type EFGI 650, BSE Bretschneider Spezialelektornik, Germany) from the aforementioned location in the Włocławek Reservoir at a depth of 0.5-1 m. The red piranha individuals were obtained from an aquarium shop. Immediately after capture, the racer goby were transported in 10-1 containers with aerated water (3-4 individuals per a container, transport time: ca. $1.5 \mathrm{~h}$ ) to $100-1$ stock tanks (8-10 individuals per tank) located in an air conditioned room at a constant temperature of $19^{\circ} \mathrm{C}$. The piranhas $(10$ individuals per tank) were kept in $200-1$ tanks at $20-21^{\circ} \mathrm{C}$ (sustained by aquarium heaters).

Water in the stock tanks with amphipods and fish was constantly oxygenated and filtered using standard aquarium filters. Every week, we exchanged ca. 30\% of the water volume. The animals were fed daily with frozen chironomid larvae. We did not observe any negative effects of transport and stocking conditions upon amphipod and fish condition and survival. The collection of fish and the experiment were conducted under permit of the Local Ethics Committee in Bydgoszcz, Poland, statement no 35/2013 from 12 December 2013.

All fish and subsamples of 100 randomly selected specimens of each amphipod species were measured with ImageJ 1.40 software (freeware by W. S. Rasband, U. S. National Institutes of Health, Bethesda, Maryland, USA, http://www.rsb.info.nih.gov/i). Mean body length of the amphipods did not differ between the species and was equal to $14.5 \mathrm{~mm}$ (min-max: 9-18.5 mm). The mean total length of the used fish also did not differ between the species and was equal to $8.5 \mathrm{~cm}$ (min-max: $7.5-10.0 \mathrm{~cm}$ ). The amphipods and chironomid larvae used as donors of the alarm substance and fish food were weighed with Radwag AS 110/C/2 laboratory scales (Radom, Poland) to estimate the signal dose.

\section{Experimental setup}

To analyse the reactions of amphipods to fish kairomones and/or alarm substances, we used a glass $\mathrm{y}$-maze constructed according to Baumgärtner et al. (2002) (Fig. 1). Gravitational inflow of water to the $\mathrm{y}$-arms was provided from two separate 40-1 source tanks containing appropriate signals or control water. The water flow in each $y$-arm was adjusted to $0.51 \mathrm{~min}^{-1}$ using ball valves, and the water level was established at $5 \mathrm{~cm}$. The outlet from the mixing zone and both inlets to the $y$-arms were covered by 1-mm mesh preventing tested animals from leaving the tank. We conducted the experiments during daytime, between ca. 9.00 and 18.00. Homogenous 


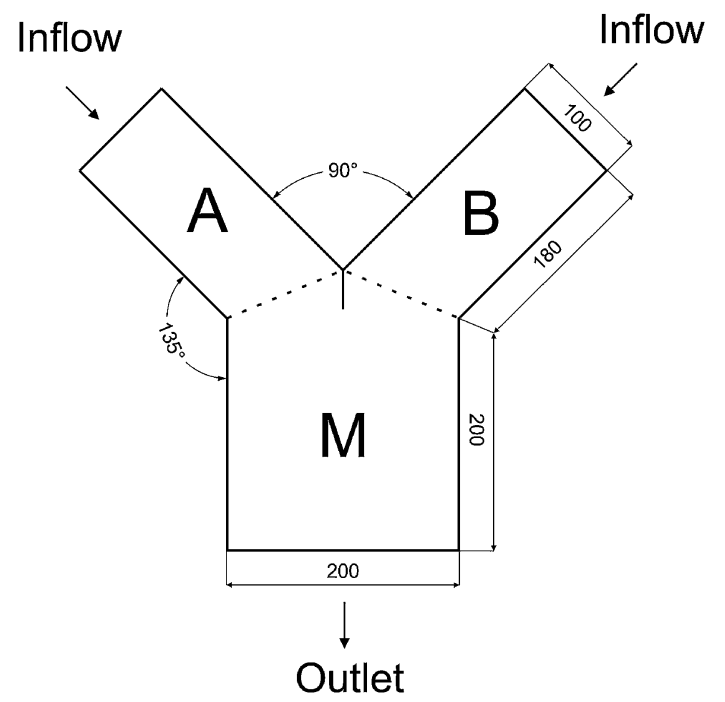

Fig. 1 Y-maze used in the alarm cue perception experiments. $A, B$ test arms to which different signals can be applied; $M$ mixing zone

indirect light conditions (520 lx) were established and controlled daily via a light meter (L-20A, Sonopan Ltd., Białystok, Poland). We conducted the experiments at a temperature of $19^{\circ} \mathrm{C}$, which commonly occurs from late spring till summer in shallow, nearshore areas of large European temperate rivers and reservoirs and is experienced by the tested species living there.

Water containing kairomones was obtained by incubation of 10 fish of a single species in a 200-L tank with fresh, filtered and aerated water for $24 \mathrm{~h}$ at $19^{\circ} \mathrm{C}$. As the fish were incubated at a high density in a closed system, they certainly provided sufficient concentration of kairomone. Predator kairomone, to be effective in the field, needs to be detected by prey at lower concentrations than those obtained under laboratory conditions. We conducted three diet variants for each predator species: (1) frozen chironomidae larvae (60-80 individuals), also used to feed amphipods; (2) 20 living individuals of $P$. robustoides per day and (3) 20 living individuals of $D$. villosus per day and additionally the fourth variant with (4) starving predators. These quantities corresponded to ca. $1.2 \mathrm{~g}$ of the total wet biomass. The fish were fed the particular source of food for 3 days before their incubation, which can be regarded as sufficient to clean their digestive tracts from the remnants of previous food (Tseitlin, 1980). They were not fed during the incubation itself to avoid cues from nonconsumed food organisms in the signal water. In the variant with starving predators, the fish were not fed for 3 days before incubation. The entire source tank was filled with water from the incubation tank. Predator densities in the closed incubation tanks used in our study were certainly higher than those found in the wild. Thus, the concentration of potential fish kairomones also exceeded the levels experienced in nature and was sufficient to trigger changes in amphipod behaviour. The control water was prepared according to the same procedure, but without adding any fish and food sources.

The alarm signals of injured prey were obtained by manual crushing of 20 amphipods or 60-80 chironomids (the same numbers as those included in the fish diet) mixed with $10 \mathrm{ml}$ of control water. Such a mixture was filtered ( $40 \mu \mathrm{m}$ gauze) and added to one of the source tanks filled earlier with control water. This resulted in the final concentration of $30 \mathrm{mg}$ of crushed prey per 11 of water in the source tank, which is similar to the doses used by Wisenden et al. (2009) and likely to elicit the amphipod response.

The water containing kairomones or alarm cues was added to the source tank immediately before each trial, ensuring the activity of the chemical signal (even in the case of its rapid degradation). Wisenden et al. (2009) showed that alarm cues from crushed Gammarus lacustris induced anti-predator responses even after $3 \mathrm{~h}$ after release.

We tested amphipods in the presence of one of the above-mentioned predation cues (two predators $\mathrm{x}$ four food variants as well as three alarm substances) in one of the $y$-arms and control water in the other. Moreover, we also conducted a test with control water in both arms of the y-maze to check for their equality.

After stabilizing the flow, we placed 10 amphipods into the mixing zone of the y-maze. Each experimental trial lasted for $35 \mathrm{~min}$, including $5 \mathrm{~min}$ for acclimation and $30 \mathrm{~min}$ of behavioural observations. The experiment was recorded using an IP video camera (SNB-6004, Samsung, South Korea) placed above the tank.

The experiment was replicated 12-15 times (replicates with an object detection rate by the video analysis software lower than $90 \%$ were dropped from the analysis) for each type of predation cue. The order of replicates was randomized among particular treatments. After each replicate, the signal and control 
arms of the y-maze were switched, and the y-maze was cleaned carefully by rinsing it several times with hot and cold tap water.

\section{Data analysis}

To analyse the recorded video material, we used Noldus Ethovision XT 10.1 software. We determined (1) percentage of time spent by amphipods in each y-maze zone (both arms and mixing zone) and (2) number of entrances to each $y$-arm. The individuals tested in a single replicate were not independent of one another and, moreover, the software sometimes switched the identities of the individuals that had touched each other. Therefore, to avoid pseudoreplication, we determined cumulative values for all 10 individuals from each replicate and divided them by 10 to obtain average values, which were used as data points in the further analysis.

As we were interested in detecting directional responses of amphipods to the signals present in particular $y$-arms, our response variables used in the data analysis were (1) difference between the percentages of time spent by amphipods in each $y$-arm and (2) difference between the numbers of amphipod entrances to each y-arm. The differences were calculated by subtracting the value measured in the control y-arm from that measured in the signal $y$-arm. Thus, negative and positive values stood for avoidance and preference, respectively. For the control treatment containing no signals, we assigned the $y$-arms as signal or control by random. We assumed that amphipods would behave similarly in both $y$-arms in the control treatment, so that the response variables would be close to 0 , whereas their response to any signal should result in a difference from the control treatment. Moreover, we analysed (3) time spent by amphipods in the mixing zone, to check if the applied signals made them move more or less often to both y-arms (which could indicate an increase or decrease in habitat exploration by amphipods, respectively).

We analysed the response variables using a twoway ANOVA with predator identity (none, goby or piranha) and predator diet/alarm source (none, chironomids, D. villosus or $P$. robustoides) as factors. We analysed the behaviour of both amphipods separately, as particular alarm/diet type cues were not equivalent for them in terms of perceiving conspecific and heterospecific signals. The data did not depart significantly from normality and homoscedasticity assumptions, as confirmed by a Shapiro-Wilk and Levene tests, respectively. We further analysed significant main effects using Tukey HSD tests and examined significant interactions with sequential Bonferronicorrected pairwise $t$ tests.

\section{Results}

Time spent in the signal and control zone

The selectivity of both amphipods for the arms of the y-maze depended only on the food/alarm source, as shown by a significant main effect of this factor in the ANOVA (Table 1A, B). D. villosus spent significantly more time in the $y$-arm with the cues obtained from crushed chironomids or conspecifics, as well as from both predators fed these types of food (Fig. 2A, B, C). The effects of chironomid and conspecific cues on time spent by $D$. villosus in the y-arms did not differ from each other (Supplementary Table S1). P. robustoides significantly avoided the predation cue obtained from injured conspecifics, as well as both fish species fed $P$. robustoides (Fig. 3A, B, C).

Time spent in the mixing zone

Time spent by amphipods in the central zone depended on the predator identity and food/alarm source, which resulted in significant predator $\mathrm{x}$ alarm interactions in the ANOVA for both tested species (Table 1C, D). $D$. villosus spent significantly less time in the mixing zone in the presence of all used signals except that emitted by piranha fed conspecifics and goby fed chironomids (Fig. 2A, B, C, Table S3). The responses to amphipod signals were stronger than those induced by chironomids in the case of alarm and racer goby cues (Table S3). Also, the responses of D. villosus to hungry predators were stronger than those to fed fish (Table S3). P. robustoides significantly reduced time spent in the mixing zone in the presence of all signals released by fish (all diet types) except red piranha fed conspecifics (Fig. 3A, B, C). The responses to amphipod signals were stronger than those induced by chironomids in the case of the racer goby cues, whereas the opposite situation took place for piranha (Table S4). 
Table 1 Two-way ANOVA of the impact of various predation cues on the behaviour of Dikerogammarus villosus (Dv) and Pontogammarus robustoides (Pr)

\begin{tabular}{|c|c|c|c|c|c|c|c|}
\hline & Species & Variable & Effect & Df & MS & $F$ & $P$ \\
\hline \multirow[t]{4}{*}{ A } & \multirow[t]{4}{*}{$\mathrm{Dv}$} & \multirow[t]{4}{*}{ Difference in time spent in both arms } & {$[\mathrm{P}]$ redator } & 2 & 0.003 & 0.142 & 0.868 \\
\hline & & & [A]larm/food & 3 & 0.324 & 14.328 & $<0.001$ \\
\hline & & & $\mathrm{P} \times \mathrm{A}$ & 6 & 0.045 & 2.003 & 0.068 \\
\hline & & & Error & 157 & 0.023 & & \\
\hline \multirow[t]{4}{*}{ B } & \multirow[t]{4}{*}{$\operatorname{Pr}$} & \multirow[t]{4}{*}{ Difference in time spent in both arms } & {$[\mathrm{P}]$ redator } & 2 & 0.019 & 1.306 & 0.274 \\
\hline & & & [A]larm/food & 3 & 0.062 & 4.171 & 0.007 \\
\hline & & & $\mathrm{P} \times \mathrm{A}$ & 6 & 0.024 & 1.612 & 0.147 \\
\hline & & & Error & 156 & 0.015 & & \\
\hline \multirow[t]{4}{*}{$\mathrm{C}$} & \multirow[t]{4}{*}{ Dv } & \multirow[t]{4}{*}{ Difference in the mixing zone occupancy } & {$[\mathrm{P}]$ redator } & 2 & 0.006 & 1.083 & 0.341 \\
\hline & & & [A]larm/food & 3 & 0.037 & 6.927 & $<0.001$ \\
\hline & & & $\mathrm{P} \times \mathrm{A}$ & 6 & 0.069 & 13.11 & $<0.001$ \\
\hline & & & Error & 157 & 0.005 & & \\
\hline \multirow[t]{4}{*}{$\mathrm{D}$} & \multirow[t]{4}{*}{$\operatorname{Pr}$} & \multirow[t]{4}{*}{ Difference in the mixing zone occupancy } & {$[\mathrm{P}]$ redator } & 2 & 0.118 & 48.673 & $<0.001$ \\
\hline & & & [A]larm/food & 3 & 0.005 & 1.98 & 0.119 \\
\hline & & & $\mathrm{P} \times \mathrm{A}$ & 6 & 0.025 & 10.406 & $<0.001$ \\
\hline & & & Error & 156 & 0.002 & & \\
\hline \multirow[t]{4}{*}{$\mathrm{E}$} & \multirow[t]{4}{*}{ Dv } & \multirow[t]{4}{*}{ Difference in the number of entrances to both arms } & {$[\mathrm{P}]$ redator } & 2 & 0.011 & 0.396 & 0.674 \\
\hline & & & [A]larm/food & 3 & 0.284 & 9.914 & $<0.001$ \\
\hline & & & $\mathrm{P} \times \mathrm{A}$ & 6 & 0.186 & 6.508 & $<0.001$ \\
\hline & & & Error & 157 & 0.029 & & \\
\hline \multirow[t]{4}{*}{$\mathrm{F}$} & \multirow[t]{4}{*}{$\operatorname{Pr}$} & \multirow[t]{4}{*}{ Difference in the number of entrances to both arms } & {$[\mathrm{P}]$ redator } & 2 & 0.016 & 0.61 & 0.545 \\
\hline & & & [A]larm/food & 3 & 0.379 & 14.247 & $<0.001$ \\
\hline & & & $\mathrm{P} \times \mathrm{A}$ & 6 & 0.091 & 3.434 & 0.003 \\
\hline & & & Error & 156 & 0.027 & & \\
\hline
\end{tabular}

Number of entrances to the signal and control zones

The number of entrances to the $y$-arms by amphipods depended on the predator identity and food/alarm source, which resulted in significant predator $\mathrm{x}$ alarm interactions in the ANOVA for both tested species (Table 1E, F).

D. villosus significantly less often entered the $y$-arm containing the signals of the racer goby fed $P$. robustoides and both hungry predators. The opposite reaction was observed to the y-arm containing the signals of the racer goby fed conspecifics and red piranha fed chironomids (Fig. 2D, E, F; Table S5).

$P$. robustoides significantly less often entered the signal y-arm containing cues of injured amphipods of both species and predators fed these types of food except the racer goby fed D. villosus (Fig. 3B; Table S6).

\section{Discussion}

Our results showed that both used species, although their common origin and feeding preferences exhibited remarkably different strategies of recognizing predation risk. Furthermore, we demonstrated that a predator cue could be used by D. villosus as a source of information about both predation risk and food resources.

In the presence of hungry predators, $D$. villosus in our study seemed to exhibit a freezing response (Sih \& Wooster, 1994; Englund, 1997), resulting in the relatively long time spent in the signal zone accompanied by the overall lower activity of animals. Thus, D. villosus used chemoreception to avoid predators, confirming the observations by Hesselschwerdt et al. (2009). Moreover, this species was also able to avoid predators without common evolutionary history. This could be explained by several mechanisms (Bourdeau 

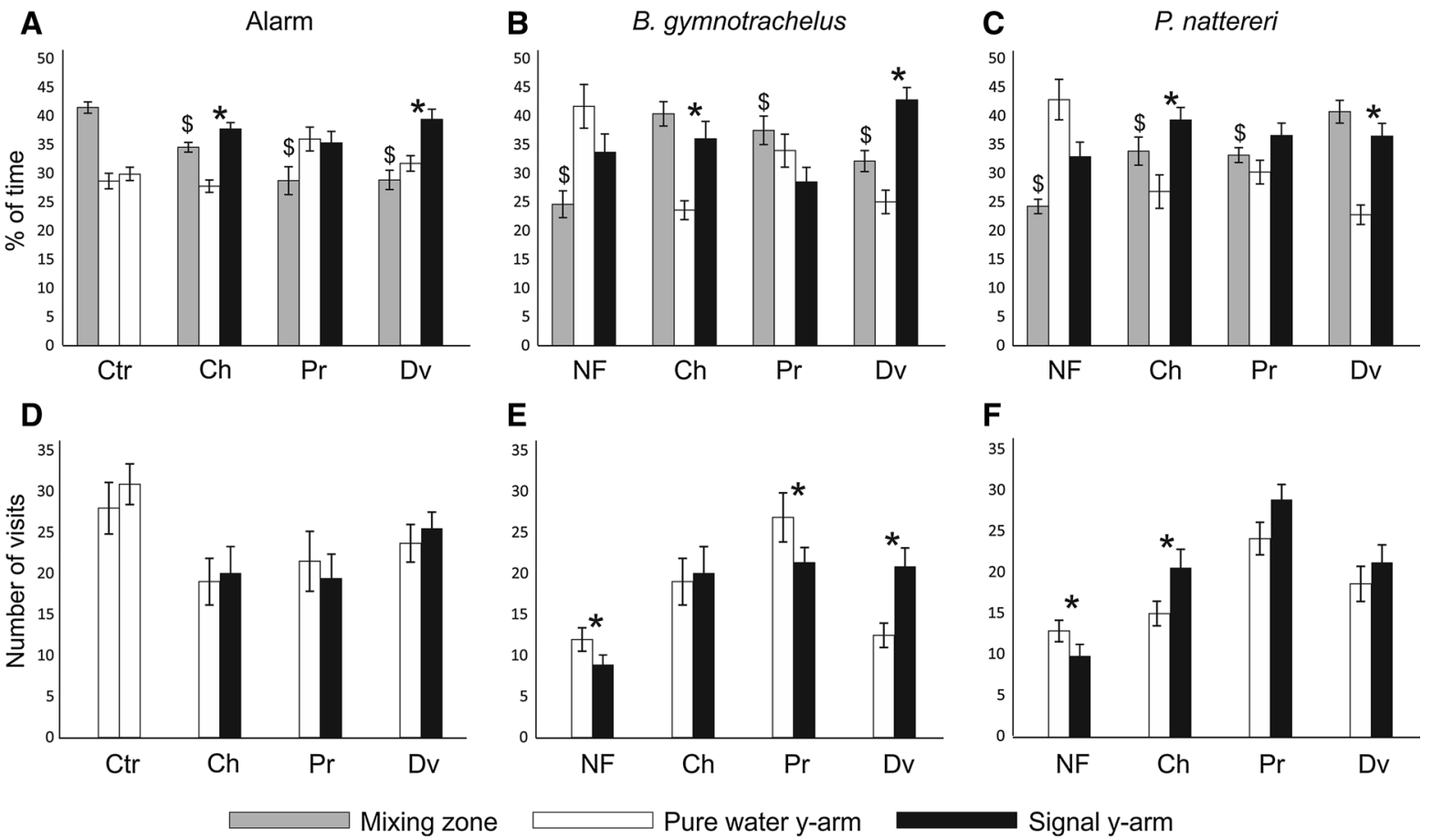

Fig. 2 Mean $( \pm$ SE) percentage occurrence times in various y-maze zones (A-C) and numbers of visits in both y-arms (DF) of Dikerogammarus villosus in the presence of different food/ alarm cues (A, D), Babka gymnotrachelus fed different diets $(\mathbf{B}$, E) and Pygocentrus nattereri fed different diets $(\mathbf{C}, \mathbf{F})$. Ctr control treatment (with pure water in both y-arms); fish diets/ alarm sources: NF, no food; $\mathrm{Ch}$, chironomids; $\mathrm{Pr}, P$. robustoides, Dv, D. villosus. The symbols above the bars indicate

et al., 2013). For example, a non-native predator cue can be similar to that emitted by co-existing predators (e.g. due to taxonomic similarity) (Ferrari et al., 2007; Sih et al., 2010) or an anti-predator response can be related to conspecific alarm signals contained in predator exudates (Chivers \& Smith, 1998). However, as the red piranha used in our study has no closely related counterparts sympatric to the Ponto-Caspian amphipods, and we observed avoidance reaction only towards hungry fish, $D$. villosus response was probably driven by other mechanisms. It could be a generalized reaction to fish, independent of species. Accordingly, von Elert \& Pohnert (2000) demonstrated that active compounds of kairomones emitted by diverse fish could be very similar if not identical, supporting this hypothesis. Such a universal response to every hungry fish could result in costly and unnecessary defence reactions to non-predatory fish. However, in temperate freshwater ecosystems, only a small part of the fish community is unable to feed on significant differences between a given treatment and the control treatment (with no signals) with regard to the difference between both y-arms (asterisks) and to the amount of time spent in the mixing zone (dollars) (pairwise sequential Bonferroni-corrected Fisher LSD tests). See supplementary Tables S1, S3 and S5 for the full details of the post hoc comparisons

amphipods (Brylińska, 2000; Kottelat \& Freyhof, 2007). Thus, such a response of $D$. villosus seems to be effective in the field.

Apart from the avoidance response to hungry predators, we have demonstrated active preferences for a range of predation cues (those associated with chironomid or conspecific exudates). Theoretically, this might be a result of a freezing response of an animal that accidentally entered a predator zone and stopped its activity after sensing the danger (Sih \& Wooster, 1994; Englund, 1997), which might have led to the aggregation of prey in the vicinity of a predator, thereby simulating a preference. However, amphipods entered and left both y-arms several times during the test, and the number of their entrances to the preferred signal zone was greater than or at least equal to the number of visits to the control y-arm. Thus, choosing the y-arm was not associated with activity reduction and did reflect a true preference of amphipods. 

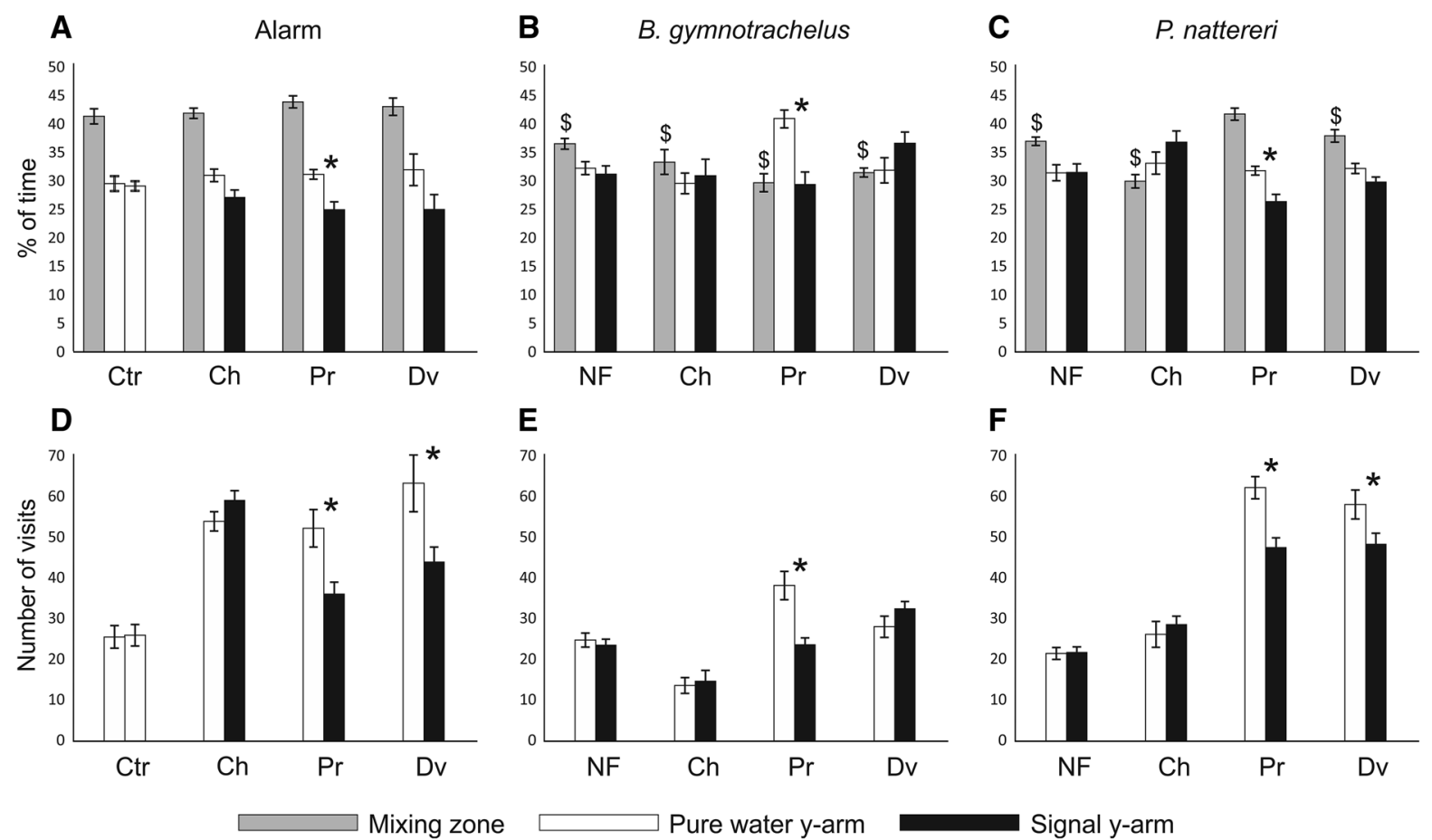

Fig. 3 Mean $( \pm S E)$ percentage occurrence times in various y-maze zones (A-C) and numbers of visits in both y-arms (DF) of Pontogammarus robustoides in the presence of different food/alarm cues (A, D), Babka gymnotrachelus fed different

Dikerogammarus villosus exhibited preferences for kairomones produced by both tested predators, but only those fed conspecifics or chironomidae larvae. This species is often regarded as an omnivore with a strong tendency to predation on macroinvertebrates (Dick \& Platvoet, 2000; Dick et al., 2002; MacNeil \& Platvoet, 2005). On the other hand, some field studies demonstrated its low trophic position, with a large share of plant food in its diet (Hellmann et al., 2015; Koester et al., 2016). Thus, the feeding habits of $D$. villosus clearly depend on a number of factors, such as the community structure (Hellmann et al., 2015) or temperature (van der Velde et al., 2009). Notwithstanding these discrepancies, it can perceive the presence of a fish not only as a danger, but also as information about potential food resources (Paterson et al., 2013), especially if the fish consumed chironomids-an important part of the diet of invasive amphipods (Bacela-Spychalska \& van der Velde, 2013). Amphipods were found to assimilate and grow better on chironomids than on plant food (Gergs \& Rothhaupt, 2008), thus chironomids seems likely to be diets (B, E) and Pygocentrus nattereri fed different diets $(\mathbf{C}, \mathbf{F})$. See Fig. 2 for the meaning of symbols and supplementary tables S2, S4 and S6 for the full details of the post hoc comparisons

their preferred food type. In such a situation, amphipods attracted to fish may benefit from feeding directly on fish faeces or from finding an area rich in their prey. Apparently, such behaviour seems to be associated with an increased risk, however, experimental data (Błońska et al., 2015) indicate that $D$. villosus is not an optimal prey item for fish, having harder exoskeleton than other freshwater amphipods, which may result in the lower predation pressure on this species (Kobak et al., 2014). In an area rich in alternative food resources, the predation pressure on amphipods can be rather low. Furthermore, D. villosus is able to utilize available anti-predator shelters, such as stones and zebra mussel beds, more efficiently than other amphipods (Kinzler \& Maier, 2006; Kobak et al., 2014). The presence of food was also found to inhibit avoidance reactions of Gammarus pulex to fish predators (Szokoli et al., 2015), confirming that food cues can constitute a stronger stimulus than predator kairomones.

Pontogammarus robustoides exhibited remarkably different anti-predator strategy, despite its common 
origin and evolution with D. villosus. Generally, it exhibited an avoidance reaction to injured amphipods of both species and to kairomones of predators fed amphipods. A particularly strong response, involving both types of behaviour, was elicited by conspecific signals. However, contrary to $D$. villosus, this species did not respond to hungry predators. A similar relationship between the predator diet and prey response was observed for other amphipods (Smith \& Webster, 2015) or fish (Roberts \& Garcia de Leaniz, 2011). Identification of predation risk based on alarm cues emitted by conspecifics and/or related species allows to avoiding responses to false signals, despite the lack of actual predation risk. Moreover, such a mechanism allows to responding efficiently to an unknown predator. An effective reaction to a predator without a common evolutionary history can be important for the survival of invasive species in new ecosystems. Furthermore, D. villosus may be perceived by other Ponto-Caspian amphipods as a potential stronger competitor and/or predator (Jermacz et al., 2015), and this might be yet another reason for the avoidance of its signals in the fish diet by $P$. robustoides.

Contrary to the behaviour of $D$. villosus, the response of $P$. robustoides to predation cues was often associated with the increase in activity, shown by the greater number of entrances to the y-arms compared to the control treatment (except the treatments with the racer goby as a predator). Amphipods could increase their activity searching for a safe shelter. P. robustoides often buries in soft substratum (e.g. sand) to protect itself from environmental dangers (Poznańska et al., 2013), including predation (Błońska et al., 2015). Such an opportunity was not provided in our design. On the other hand, D. villosus can efficiently cling to hard or mesh surfaces (BacelaSpychalska et al., 2013), and it was easier for this species to find a suitable shelter in the y-maze (e.g. in the corners or on the mesh securing the outlets).

In many studies (Andersson et al., 1986; Wudkevich et al., 1997; Jermacz et al., 2015), amphipods responded to predator kairomones by reduction of their movement activity to avoid detection. Such a response is usually associated with indirect predator effects, such as the limitation of foraging time and mate searching. During our tests, both species significantly reduced time spent in the mixing zone in which they were initially placed, actively moving to the $y$-arms several times in all treatments. Such a reaction is related with active responses to the applied signals, leading to the occupation of a zone with the minimum or maximum concentration of the cue in the case of avoidance or preference, respectively. Amphipod activity and switching between the zones can be also increased by the fact that they only perceived chemical cues in the y-maze, but could not find real sources of these cues (i.e. no fish, food or heterospecific amphipods were present directly in the $y$-arms). Nevertheless, differences in the occurrence time and/ or numbers of visits between the $y$-arms depending on the quality of the applied signal clearly show that the amphipods responded directionally to the signal source and point to their preference for or avoidance of the cue.

In our study, the differences between responses triggered by both predator species were clearly minor compared to the effects of predator diets. Even though some differences in amphipod responses to both predators were manifested in the time spent in the mixing zone (Tables S3-4) and (less often) in the difference in the numbers of visits between the $y$-arms (Tables S5-6), both amphipods clearly responded to both fish species, exhibiting a preference or avoidance regardless of their origin. Moreover, their responses to each predator evidently differed depending on the quality of the fish diet. Contrary to our findings, Paterson et al. (2013) in their meta-analysis of responses of aquatic invertebrates to predator cues concluded that predator diet does not influence antipredatory behaviour of prey. This discrepancy can be accounted for by the peculiarity of the species tested in our study. First, both our species are omnivorous, which makes their ability to detect additional foodrelated signals in the predator scent particularly adaptive. Second, they are highly invasive, and this trait may be enhanced by the ability to respond specifically to the diet of an unknown predator. This speculation cannot be fully resolved without a direct comparison to non-invasive species. However, Paterson et al. (2013) has demonstrated that such differentiation by aquatic invertebrates is an exception, rather than a rule. On the other hand, different responses of prey to various predation cues, i.e. predator kairomones and conspecific alarm substances were also observed in the zebra mussel Dreissena polymorpha (Czarnołęski et al., 2010; Kobak et al., 2010), which is a highly invasive species (Karatayev et al., 2002). 
Thus, perhaps this trait might be associated with the invasiveness. Notwithstanding these doubts, the ability to base anti-predator responses on a predator diet, rather than on its identity, is certainly beneficial for species occupying novel areas and often encountering unknown communities. On the other hand, another amphipod, G. pulex, regarded as less invasive and less carnivorous than the species studied here (Grabowski et al., 2007), did select between two fish species posing different levels of danger (Szokoli et al., 2015).

Freshwater communities are much more heterogeneous and isolated from one another than terrestrial or marine systems (Cox \& Lima, 2006), which increases the possibility of encountering novel predators and prey species by migrating organisms. Moreover, prey tend to suffer more from unknown predators than from native enemies (Salo et al., 2007). Therefore, the ability to respond adequately to unknown predators can be particularly important for freshwater species and strongly increase their invasive potential. Such species can become particularly successful invaders and contribute to the threats posed by invasive organisms to the world's biodiversity (Clavero \& Garcia-Berthou, 2005; Simberloff \& Vitule, 2014).

To our knowledge, a positive interaction in the preypredator system shown in our study for $D$. villosus has never been found before. Wisenden et al. (2009) observed a reversed response of G. lacustris to crushed conspecifics (an avoidance of a 3-h-old exudate and a preference for a 6-h-old exudate), showing that a food signal persists longer than an alarm cue. However, in our study, the amphipods were attracted to fresh cues, prepared directly before the test, so they exhibited no avoidance at all. This phenomenon reveals unknown abilities of the studied amphipods, which can facilitate their survival and colonization.

In conclusion, the results of our study indicate that Ponto-Caspian invasive amphipods use chemoreception to detect predation risk as well as food resources. We found that the predator diet was an important factor used in the recognition of predators and triggering the prey response. Moreover, amphipods may even prefer the vicinity of predators under some conditions. This uncommon behaviour suggests strong inclination to search for feeding grounds, even despite of the increased risk of predation. Thus, omnivorous prey partly sharing their diet with a top predator may benefit from its presence if the predation risk is not high (for instance: other types of food are preferred by the top predator) and an additional signal of the vicinity of abundant food is carried by the top predator.

Our results show the importance of biotic interactions in community functioning. The presence or absence of particular predators and prey species may strongly modify the performance of organisms, including their behaviour, competitive abilities, feeding preferences and survival. For alien species, this may greatly affect the possibility of their invasion success as well as their environmental impact. It is likely that species which can correctly recognize and interpret the risk and benefit associated with the presence of other organisms would be more efficient in expanding into new areas and would exert stronger impact on local communities. Such relationships between alien species and the environment, as well as their implications for conservation issues, are difficult to predict without comprehensive knowledge of their interactions with other members of the community, including those re-created in a new place with other aliens coming from the same region and those formed anew with local taxa.

Acknowledgments Our study was supported by the Grant of the National Science Centre No. 2013/09/N/NZ8/03191.

Open Access This article is distributed under the terms of the Creative Commons Attribution 4.0 International License (http:// creativecommons.org/licenses/by/4.0/), which permits unrestricted use, distribution, and reproduction in any medium, provided you give appropriate credit to the original author(s) and the source, provide a link to the Creative Commons license, and indicate if changes were made.

\section{References}

Åbjörnsson, K., B. Wagner, A. Axelsson, R. Bjerselius \& K. H. Olsen, 1997. Responses of Acilius sulcatus (Coleoptera: Dytiscidae) to chemical cues from perch (Perca fluviatilis). Oecologia 111: 166-171.

Åbjörnsson, K., L.-A. Hansson \& C. Brönmark, 2004. Responses of prey from habitats with different predator regimes: local adaptation and heritability. Ecology 85 : 1859-1866.

Andersson, K. G., C. Brönmark, J. Herrmann, B. Malmqvist, C. Otto, P. Sjörström \& C. Bronmark, 1986. Presence of sculpins (Cottus gobio) reduces drift and activity of Gammarus pulex (Amphipoda). Hydrobiologia 133: 209-215.

Bacela-Spychalska, K. \& G. van der Velde, 2013. There is more than one "killer shrimp": trophic positions and predatory abilities of invasive amphipods of Ponto-Caspian origin. Freshwater Biology 58: 730-741. 
Bacela-Spychalska, K., M. Grabowski, T. Rewicz, A. Konopacka \& R. Wattier, 2013. The "killer Shrimp" Dikerogammarus villosus (crustacea, amphipoda) invading alpine lakes: overland transport by recreational boats and scuba-diving gear as potential entry vectors? Aquatic Conservation: Marine and Freshwater Ecosystems 23: 606-618.

Banks, P. B. \& C. R. Dickman, 2007. Alien predation and the effects of multiple levels of prey naiveté. Trends in Ecology \& Evolution 22: 229-230.

Baumgärtner, D., A. D. Jungbluth, U. Koch \& E. von Elert, 2002. Effects of infochemicals on microhabitat choice by the freshwater amphipod Gammarus roeseli. Archiv für Hydrobiologie 155: 353-367.

Baumgärtner, D., U. Koch \& K.-O. Rothhaupt, 2003. Alteration of kairomone-induced antipredator response of the freshwater amphipod Gammarus roeseli by sediment type. Journal of Chemical Ecology 29: 1391-1401.

Beermann, J., J. T. A. Dick \& M. Thiel, 2015. Social recognition in amphipods: an overview. In Aquiloni, L. \& E. Tricarico (eds), Social Recognition in Invertebrates. Springer International Publishing, Cham: 85-100.

Bernot, R. J. \& A. M. Turner, 2001. Predator identity and traitmediated indirect effects in a littoral food web. Oecologia 129: 139-146.

Błońska, D., J. Grabowska, J. Kobak, Ł. Jermacz \& K. BacelaSpychalska, 2015. Feeding preferences of an invasive Ponto-Caspian goby for native and non-native gammarid prey. Freshwater Biology 60: 2187-2195.

Bourdeau, P. E., K. L. Pangle, E. M. Reed \& S. D. Peacor, 2013. Finely tuned response of native prey to an invasive predator in a freshwater system. Ecology 94: 1449-1455.

Brandner, J., K. Auerswald, A. F. Cerwenka, U. K. Schliewen \& J. Geist, 2013. Comparative feeding ecology of invasive Ponto-Caspian gobies. Hydrobiologia 703: 113-131.

Brönmark, C. \& L. Hansson, 2000. Chemical communication in aquatic systems: an introduction. Oikos 88: 103-109.

Brylińska, M., 2000. Ryby sodkowodne Polski. PWN Wydawnictwo Naukowe, Warszawa.

Chivers, D. P. \& R. J. F. Smith, 1998. Chemical alarm signalling in aquatic predator-prey systems: a review and prospectus. Ecoscience 5: 338-352.

Chivers, D. P., B. D. Wisenden \& R. J. F. Smith, 1996. Damselfly larvae learn to recognize predators from chemical cues in the predator's diet. Animal Behaviour 52: 315-320.

Clavero, M. \& E. Garcia-Berthou, 2005. Invasive species are a leading cause of animal extinctions. Trends in Ecology \& Evolution 20: 110.

Clinchy, M., M. J. Sheriff \& L. Y. Zanette, 2013. Predatorinduced stress and the ecology of fear. Functional Ecology 27: $56-65$

Cox, J. G. \& S. L. Lima, 2006. Naivete and an aquatic-terrestrial dichotomy in the effects of introduced predators. Trends in Ecology and Evolution 21: 674-680.

Czarnołęski, M., T. Müller, K. Adamus, G. Ogorzelska \& M. Sog, 2010. Injured conspecifics alter mobility and byssus production in zebra mussels Dreissena polymorpha. Fundamental and Applied Limnology/Archiv für Hydrobiologie 176: 269-278.

Dick, J. T. A. \& D. Platvoet, 2000. Invading predatory crustacean Dikerogammarus villosus eliminates both native and exotic species. Proceedings of the Royal Society B: Biological Sciences 267: 977-983.

Dick, J. T. A., D. Platvoet \& D. W. Kelly, 2002. Predatory impact of the freshwater invader Dikerogammarus villosus (Crustacea: Amphipoda). Canadian Journal of Fisheries and Aquatic Sciences 59: 1078-1084.

Dunn, A. M., J. T. A. Dick \& M. J. Hatcher, 2008. The less amorous Gammarus: predation risk affects mating decisions in Gammarus duebeni (Amphipoda). Animal Behaviour 76: 1289-1295.

Englund, G., 1997. Importance of spatial scale and prey movements in predator caging experiments. Ecology 78: 2316-2325.

Ferrari, M. C., A. Gonzalo, F. Messier \& D. P. Chivers, 2007. Generalization of learned predator recognition: an experimental test and framework for future studies. Proceedings of the Royal Society B: Biological Sciences 274: 1853-1859.

Gergs, R. \& K.-O. Rothhaupt, 2008. Feeding rates, assimilation efficiencies and growth of two amphipod species on biodeposited material from zebra mussels. Freshwater Biology 53: 2494-2503.

Gliwicz, Z. M., 1994. Relative significance of direct and indirect effects of predation by planktivorous fish on zooplankton. Hydrobiologia 272: 201-210.

Grabowski, M., K. Bacela, A. Konopacka, K. Bącela \& A. Konopacka, 2007. How to be an invasive gammarid (Amphipoda: Gammaroidea) - comparison of life history traits. Hydrobiologia 590: 75-84.

Haddaway, N. R., D. Vieille, R. J. G. Mortimer, M. Christmas \& A. M. Dunn, 2014. Aquatic macroinvertebrate responses to native and non-native predators. Knowledge and Management of Aquatic Ecosystems 415: 10.

Hellmann, C., S. Worischka, E. Mehler, J. Becker, R. Gergs \& C. Winkelmann, 2015. The trophic function of Dikerogammarus villosus (Sowinsky, 1894) in invaded rivers: a case study in the Elbe and Rhine. Aquatic Invasions 10: 385-397.

Hesselschwerdt, J., S. Tscharner, J. Necker \& K. M. Wantzen, 2009. A local gammarid uses kairomones to avoid predation by the invasive crustaceans Dikerogammarus villosus and Orconectes limosus. Biological Invasions 11: 2133-2140.

Jażdżewski, K., A. Konopacka \& M. Grabowski, 2002. Four Ponto-Caspian and one American gammarid species (Crustacea, Amphipoda) invading Polish waters. Contributions to Zoology 71: 115-122.

Jermacz, Ł., A. Dzierżyńska, T. Kakareko, M. Poznańska \& J. Kobak, 2015. The art of choice: predation risk changes interspecific competition between freshwater amphipods. Behavioral Ecology 26: 656-664.

Kakareko, T., J. Zbikowski \& J. Zytkowicz, 2005. Diet partitioning in summer of two syntopic neogobiids from two different habitats of the lower Vistula River, Poland. Journal of Applied Ichthyology Journal of Applied Ichthyology 21: 292-295.

Karatayev, A. Y., L. E. Burlakova \& D. K. Padilla, 2002. Impacts of zebra mussels on aquatic communities and their role as ecosystem engineers. In Leppäkoski, E., S. Gollasch \& S. Olenin (eds), Invasive Aquatic Species of Europe. Distribution, Impacts and Management. Springer, Dordrecht: 433-446. 
Kinzler, W. \& G. Maier, 2006. Selective predation by fish: a further reason for the decline of native gammarids in the presence of invasives? Journal of Limnology 65: 27-34.

Kobak, J., Ł. Jermacz \& D. Płąchocki, 2014. Effectiveness of zebra mussels to act as shelters from fish predators differs between native and invasive amphipod prey. Aquatic Ecology 48: 397-408.

Kobak, J., T. Kakareko \& M. Poznańska, 2010. Changes in attachment strength and aggregation of zebra mussel, Dreissena polymorpha in the presence of potential fish predators of various species and size. Hydrobiologia 644: 195-206.

Koester, M., B. Bayer \& R. Gergs, 2016. Is Dikerogammarus villosus (Crustacea, Gammaridae) a "killer shrimp" in the River Rhine system? Hydrobiologia 768: 299-313.

Kottelat, M. \& J. Freyhof, 2007. Handbook of European Freshwater Fishes. Publications Kottelat, Cornol.

Lima, S. L. \& L. M. Dill, 1990. Behavioral decisions made under the risk of predation: a review and prospectus. Canadian Journal of Zoology NRC Research Press 68: 619-640.

MacNeil, C. \& D. Platvoet, 2005. The predatory impact of the freshwater invader Dikerogammarus villosus on native Gammarus pulex (Crustacea: Amphipoda); influences of differential microdistribution and food resources. Journal of Zoology 267: 31-38.

MacNeil, C., J. T. A. Dick \& R. W. Elwood, 1997. The trophic ecology of freshwater Gammarus spp. (Crustacea: Amphipoda): Problems and perspectives concerning the functional feeding group concept. Biological Reviews 72: 349-364.

Paterson, R. A., D. W. Pritchard, J. T. A. Dick, M. E. Alexander, M. J. Hatcher \& A. M. Dunn, 2013. Predator cue studies reveal strong trait-mediated effects in communities despite variation in experimental designs. Animal Behaviour 86: 1301-1313.

Pennuto, C. \& D. Keppler, 2008. Short-term predator avoidance behavior by invasive and native amphipods in the Great Lakes. Aquatic Ecology 42: 629-641.

Poznańska, M., T. Kakareko, M. Krzyżyński \& J. Kobak, 2013. Effect of substratum drying on the survival and migrations of Ponto-Caspian and native gammarids (Crustacea: Amphipoda). Hydrobiologia 700: 47-59.

Radloff, F. G. T. \& J. T. Du Toit, 2004. Large predators and their prey in a southern African savanna: a predator size determines its prey size range. Journal of Animal Ecology 73: 410-423.

Relyea, R. A., 2008. How prey respond to combined predators: a review and an empirical test. Ecology 84: 1827-1839.

Rewicz, T., M. Grabowski, C. MacNeil \& K. Bącela-Spychalska, 2014. The profile of a "perfect" invader - the case of killer shrimp. Aquatic Invasions 9: 267-288.

Roberts, L. J. \& C. Garcia de Leaniz, 2011. Something smells fishy: predator-naïve salmon use diet cues, not kairomones, to recognize a sympatric mammalian predator. Animal Behaviour 82: 619-625.

Salo, P., E. Korpimaki, P. B. Banks, M. Nordstrom \& C. R. Dickman, 2007. Alien predators are more dangerous than native predators to prey populations. Proceedings of the Royal Society B: Biological Sciences 274: 1237-1243.

Schoeppner, N. M. \& R. A. Relyea, 2009. Interpreting the smells of predation: how alarm cues and kairomones induce different prey defences. Functional Ecology 23: 1114-1121.

Sih, A. \& D. E. Wooster, 1994. Prey behavior, prey dispersal, and predator impacts on stream prey. Ecology 75: 1199-1207.

Sih, A., D. I. Bolnick, B. Luttbeg, J. L. Orrock, S. D. Peacor, L. M. Pintor, E. Preisser, J. S. Rehage \& J. R. Vonesh, 2010. Predator-prey naïveté, antipredator behavior, and the ecology of predator invasions. Oikos 119: 610-621.

Simberloff, D. \& J. R. S. Vitule, 2014. A call for an end to calls for the end of invasion biology. Oikos 123: 408-413.

Smith, L. A. \& M. M. Webster, 2015. Gammarus pulex show a grouping response to conspecific injury cues but not to predator kairomones. Behavioral Ecology 26: 1188-1195.

Szokoli, F., C. Winkelmann, T. U. Berendonk \& S. Worischka, 2015. The effects of fish kairomones and food availability on the predator avoidance behaviour of Gammarus pulex. Fundamental and Applied Limnology 186: 249-258.

Tseitlin, V., 1980. Duration of gastric digestion in fishes. Marine Ecology Progress Series 2: 277-280.

van der Velde, G., R. S. E. W. Leuven, D. Platvoet, K. Bącela, M. A. J. Huijbregts, H. W. M. Hendriks \& D. Kruijt, 2009. Environmental and morphological factors influencing predatory behaviour by invasive non-indigenous gammaridean species. Biological Invasions 11: 2043-2054.

von Elert, E. \& G. Pohnert, 2000. Predator specificity of kairomones in diel vertical migration of Daphnia: a chemical approach. Oikos 88: 119-128.

Weber, A., 2003. More than one "fish kairomone"? Perch and stickleback kairomones affect Daphnia life history traits differently. Hydrobiologia 498: 143-150.

Werner, E. E. \& S. D. Peacor, 2003. A review of trait-mediated indirect interactions in ecological communities. Ecology 84: 1083-1100.

Wisenden, B. D., D. P. Chivers \& R. J. F. Smith, 1997. Learned recognition of predation risk by Enallagma damselfly larvae (Odonata, Zygoptera) on the basis of chemical cues. Journal of Chemical Ecology 23: 137-151.

Wisenden, B. D., M. L. Rugg, N. L. Korpi \& L. C. Fuselier, 2009. Lab and field estimates of active time of chemical alarm cues of a cyprinid fish and an amphipod crustacean. Behaviour 146: 1423-1442.

Wudkevich, K., B. D. Wisenden, D. P. Chivers \& R. J. F. Smith, 1997. Reactions of Gammarus lacustris to chemical stimuli from natural predators and injured conspecifics. Journal of Chemical Ecology 23: 1163-1173.

Yoshida, T., L. E. Jones, S. P. Ellner, G. F. Fussmann \& N. G. Hairston, 2003. Rapid evolution drives ecological dynamics in a predator-prey system. Nature 424: 303-306.

Zuharah, W. F. \& P. J. Lester, 2010. Are exotic invaders less susceptible to native predators? A test using native and exotic mosquito species in New Zealand. Population Ecology 53: 307-317. 